UM-ChE-98/605

\title{
Universality of the excess number of clusters and the crossing probability function in three-dimensional percolation
}

\author{
Christian D. Lorenz ${ }^{\#}$ and Robert M. Ziff ${ }^{\dagger}$ \\ University of Michigan, Ann Arbor, MI 48109-2136
}

(March 6, 2022)

\begin{abstract}
Extensive Monte-Carlo simulations were performed to evaluate the excess number of clusters and the crossing probability function for three-dimensional percolation on the simple cubic (s.c.), face-centered cubic (f.c.c.), and bodycentered cubic (b.c.c.) lattices. Systems $L \times L \times L^{\prime}$ with $L^{\prime}>>$ were studied for both bond (s.c., f.c.c., b.c.c.) and site (f.c.c.) percolation. The excess number of clusters $\tilde{b}$ per unit length was confirmed to be a universal quantity with a value $\tilde{b} \approx 0.412$. Likewise, the critical crossing probability in the $L^{\prime}$ direction, with periodic boundary conditions in the $L \times L$ plane, was found to follow a universal exponential decay as a function of $r=L^{\prime} / L$ for large $r$. Simulations were also carried out to find new precise values of the critical thresholds for site percolation on the f.c.c. and b.c.c. lattices, yielding $p_{c}$ (f.c.c. $)=0.1992365 \pm 0.0000010, p_{c}$ (b.c.c. $)=0.2459615 \pm 0.0000010 . \mathrm{We}$ also report the value $p_{c}$ (s.c.) $=0.3116080 \pm 0.0000004$ for site percolation. PACS numbers(s): 64.60Ak, 05.70.Jk
\end{abstract}




\section{INTRODUCTION}

The standard percolation model [1] involves the random occupation of sites or bonds of a regular lattice. At a critical occupation probability $p_{c}$, the mean size of clusters of occupied sites becomes infinite, while the number of clusters $n(p)$ per site or per unit volume remains finite with $n_{c}=n\left(p_{c}\right)$.

The value of $n_{c}$ depends on the microscopic characteristics of each system, and because of this it is a non-universal quantity. For two-dimensional (2d) systems, precise numerical values of $n_{c}$ for bond and site percolation on the square and triangular lattices were found by Ziff, Finch, and Adamchik [2], whose results for bond percolation confirmed the theoretical predictions of Temperley and Lieb [3] and Baxter, Temperley, and Ashley [4]. In 3d, there are no theoretical predictions for $n_{c}$, and its values for different systems apparently have not been reported in literature.

In Ref. [Q] $\left.n_{c}\right)$, with $r=L^{\prime} / L=$ fixed, where $n\left(L, L^{\prime}\right)$ is the number of clusters per unit area in a finite system of size $L \times L^{\prime}$ with periodic boundary conditions, is a universal quantity that depends only upon aspect ratio $r$. (Note that in [2], the authors defined $n$ as clusters per site rather than per unit area, but the result for $b$ is the same.) This universality is consistent with the arguments of Privman and Fisher [5], and has also been discussed by Aharony and Stauffer [6] and by Müller [7] for the Ising model. Kleban and Ziff [8] introduced an excess number per unit length $\tilde{b} \equiv \lim _{r \rightarrow \infty} b(r) / r=\lim _{L \rightarrow \infty} L^{2}\left(n\left(L, L^{\prime}\right)-n_{c}\right)$ for long cylindrical systems $L^{\prime}>$ > and derived exact results for both $b(r)$ and $\tilde{b}$ in 2 d systems. Again, however, no theoretical predictions for $b$ in $3 \mathrm{~d}$ exist.

In this paper, we determine $n_{c}$ and $\tilde{b}$ for various $3 \mathrm{~d}$ rectangular solid systems of dimensions $L \times L \times L^{\prime}$ with $L^{\prime}>$ > We consider bond percolation on the simple cubic (s.c.), body-centered cubic (b.c.c.), and face-centered cubic (f.c.c.) lattices, and site percolation on the f.c.c. lattice.

A prerequisite to finding the value of $n_{c}$ for each of these systems is knowing the critical 
occupational probability $p_{c}$ to high accuracy. Previously, accurate values were found for bond percolation on all three lattices and site percolation on the s.c. lattice only, as summarized in Table $\square$. To round out these values, we carried out simulations to determine $p_{c}$ for site percolation on the b.c.c. and f.c.c. lattices to high accuracy — although we used only the latter in the study of the excess cluster number, since the universality was clearly confirmed with the four systems that we studied. In another work [9], we have studied site percolation on the s. c. lattice, and report this result in Table 1 also.

The simulations for finding $n_{c}$ were also used to study the critical crossing probability for the three-dimensional systems. The crossing probability function $\pi(\Gamma)$ of a system of shape $\Gamma$ gives the probability that at least one cluster connects two disjoint pieces of the boundary $\partial \Gamma$, and has been of much interest lately following the realization that it is a fundamental, universal property of percolation, independent of the underlying lattice type, and subject to conformal invariance [10 13]. In 2d, Cardy [11] derived an explicit expression for the vertical crossing probability $\pi_{v}$ of rectangular systems $L \times L^{\prime}$, with open boundaries in the horizontal direction, and Watts [14] derived an expression for the probability of vertical but not horizontal crossing for this system. The $\pi_{v}$ for $2 \mathrm{~d}$ systems with periodic (and other) boundary conditions was studied by Hovi and Aharony 15. A number of systems were also studied by various groups including $\mathrm{Hu}$ et al. [16], Hsu et al. [17], Gropengiesser [18], and Vicsek and Kertész [19]. In 3d, work has been restricted to simple cubical boundaries $L \times L \times L$, with crossing studied between two opposite planes and various boundary conditions on the sides [16,20,21.

Here we find $\pi_{v}$ for the $L \times L \times L^{\prime}$ systems for all $L^{\prime}$ by measuring the distribution of the maximum height of clusters connected to the base of the rectangular system. (A similar method was used in [22 for 2d systems.) We consider crossing in the $L^{\prime}$ direction for systems with periodic boundary conditions in the $L \times L$ plane, and show that $\pi_{v}$ is a universal function of $r=L^{\prime} / L$ for large $L$.

In the following three sections we report on the determination of the new values of $p_{c}$, the determination of $n_{c}$ and $\tilde{b}$, and the determination of $\pi_{v}(r)$. The results are summarized 
and discussed further in the conclusions section.

\section{PERCOLATION THRESHOLDS}

Precise values for the thresholds for bond percolation on all three lattices, and for site percolation on the s.c. lattice, have been found elsewhere. Here we also determine accurate values for site percolation on the f.c.c. and b.c.c. lattices. A summary of our results and other recent results is given in Table [1.

The procedure we used to find $p_{c}$ was similar to that we used for bond percolation in [23]. We grew individual clusters by a Leath-type algorithm and identified the critical point using an epidemic scaling analysis. A virtual lattice of $2048^{3}$ sites was simulated, using the block-data method first described in [24]. There were only two minor changes made to the simulation of [23] so that it could be used to study site percolation. First, as the clusters were grown, the sites were either occupied with a probability, $p$, or left vacant with a probability, $1-p$. If a site was determined to be vacant, then (unlike in bond percolation) it was never revisited as a potential growing site. The other difference is the cut-off for the growth of these clusters was set to $2^{19}(524,288)$ wetted sites, as opposed to $2^{20}(1,048,576)$ and $2^{21}(2,097,152)$ in ref. [23].

The simulation yielded the fraction of clusters $P(s, p)$ that grew to a size greater than or equal to $s$ sites. When $p$ is near $p_{c}$, one expects $P(s, p)$ to behave as

$$
P(s, p) \sim A s^{2-\tau} f\left(\left(p-p_{c}\right) s^{\sigma}\right) \approx A s^{2-\tau}\left[1+C\left(p-p_{c}\right) s^{\sigma}+\ldots\right]
$$

where $\tau$ and $\sigma$ are universal exponents [25]. Here we assumed the values $\tau=2.189$ and $\sigma=0.445$, consistent with other $3 \mathrm{~d}$ work [23,26,27]. As in [23], plots of $s^{\tau-2} P(s, p)$ vs. $s^{\sigma}$ for site percolation of the b.c.c. and f.c.c. lattices were used to find the value of the percolation threshold, which corresponds to horizontal behavior for large $L$ on such a plot. In all, we generated $1.5 \times 10^{7}$ clusters for the f.c.c. lattice and $2.2 \times 10^{7}$ for the b.c.c. lattice for a range of values of $p$ requiring several weeks of workstation computer time. The results are plotted in Fig. 1 and imply the following values for the critical thresholds: 


$$
\begin{aligned}
& p_{c}(\text { b.c.c. })=0.2459615 \pm 0.0000010 \\
& p_{c}(\text { f.c.c. })=0.1992365 \pm 0.0000010
\end{aligned}
$$

These results were consistent with (and more than 1000 times more precise than) previous work, as shown in Table [.

\section{VALUES OF $N_{C}$ AND THE FINITE-SIZE CORRECTION $\tilde{B}$}

Using the values of the critical thresholds given in Table \&, we carried out simulations to measure the number of clusters for bond percolation on each of the three-dimensional lattices and site percolation on the f.c.c. lattice. (We did not consider site percolation on the s.c. and b.c.c. lattice in this calculation.) Clusters were grown successively from every unvisited site by a growth algorithm 28] on a three-dimensional square bar, $L \times L \times L^{\prime}$ with $L^{\prime}>>L$. Periodic boundary conditions were assumed in each horizontal plane. (Here, vertical is taken to be the $L^{\prime}$ direction). The first cluster was started in the upper lefthand corner of the first plane $(z=0)$ at the point $(0,0,0)$. From this corner, a cluster was

grown to the nearest neighboring sites as defined for each system by the unit vectors in [23], occupying the connecting bonds or neighboring sites with a probability, $p_{c}$, and leaving them unoccupied with a probability, $1-p_{c}$. After the first cluster was grown, a new cluster was seeded from the first unoccupied site in the left-most column, and grown until it died. After all sites of the first plane were tested, the growing plane was moved to $z=1$, and so on. Because the previous planes were completely occupied, their data could be discarded and the memory recycled. Furthermore, the clusters never extended up to a plane of distance $z=32 L$ from the growing plane. As a consequence, a system of size $L \times L \times 32 L$ could be used to effectively simulate a $L \times L \times \infty$ system by wrapping around in the third direction.

We ran simulations to $L^{\prime}=2 \times 10^{9}$, with $L=4,5,6,7,8,10$, and 12 for the s.c. lattice, $L=4,6,8,10$ and 12 for the f.c.c. lattice (both site and bond), and $L=4,6,8$, 10,12 , and 16 for the b.c.c. lattice. In total, we grew about $1.06 \times 10^{12}$ clusters for the b.c.c. lattice, $1.88 \times 10^{12}$ clusters for the s.c. lattice, $1.44 \times 10^{12}$ clusters for bond percolation 
on the f.c.c. lattice, and $3.32 \times 10^{11}$ for site percolation on the f.c.c. lattice, which required several additional months of computer time.

In Figure 2, we display a representative $4 \times 4$ plane of each of the three lattices, showing how the lattices were oriented in our simulations and how the unit dimension was defined. The darkened circles represent active sites in the current plane, and the empty circles represent active sites that are in the neighboring planes. The solid lines are bonds which lie within the current plane and the dashed lines represent bonds which connect the displayed plane to the neighboring planes. For modeling the s.c. lattice, the plane shown in Figure $2(a)$ is repeated for the whole length of the cylinder, while for the other two lattices, the plane shown in the figure is repeated on every other plane. In the case of the s.c. lattice, all of the available sites in the plane are considered active, for the f.c.c. lattice, only half of the underlying cubic-lattice sites are active, and for the b.c.c. lattice, only a quarter of the cubic-lattice sites are active. Note that the unit dimension that we define for the b.c.c. and f.c.c. lattices is neither the unit cell dimension nor the nearest-neighbor distance, but half of the unit cell dimension.

Now, for a finite system of volume $V$ with periodic boundary conditions, analogous to what was found in [2] for $2 \mathrm{~d}$, we expect

$$
n=n_{c}+\frac{b}{V}+\frac{c}{V^{2}}+\ldots
$$

where $b$, representing the excess number of clusters in this finite system, is universal, a function of the shape only. Here we studied $L \times L \times L^{\prime}$ systems, where $L^{\prime}>>L$, with the volume given by $V=L^{2} L^{\prime}$. For systems of this shape, we expect the excess number of clusters per unit length $b /\left(L^{\prime} / L\right)$ to be a constant $\tilde{b}$, i.e.,

$$
b \sim \tilde{b} L^{\prime} / L
$$

for $L^{\prime}>>L$. Likewise, we write $c \sim \tilde{c}\left(L^{\prime} / L\right)^{2}$. Then it follows from (3) that

$$
n=n_{c}+\frac{\tilde{b}}{L^{3}}+\frac{\tilde{c}}{L^{6}}+\ldots
$$


Both $b$ and $\tilde{b}$ are functions of the system shape only and are universal quantities, but $c$ and $\tilde{c}$ vary from system to system and are not universal. Eq. (5) implies that $n_{c}$ can be found from a plot of $n$ vs. $1 / L^{3}$, as shown in Figure 3 for our data from the b.c.c. lattice. The values of $n_{c}$, which are shown in units of number of clusters per unit volume as defined in Fig. 2 for the various lattices, are given in Table [1]. These values can be converted to units of number of clusters per site by taking into account that the s.c., f.c.c., and b.c.c. lattices have $1,1 / 2$, and $1 / 4$ sites per unit volume, respectively.

Equation (5) can be rearranged as

$$
\left(n-n_{c}\right) L^{3}=\tilde{b}+\frac{\tilde{c}}{L^{3}}+\ldots
$$

Therefore, once $n_{c}$ is determined, $\tilde{b}$ and $\tilde{c}$ can be found from a plot of $\left(n-n_{c}\right) L^{3}$ vs. $1 / L^{3}$. Figure 4 shows this plot for the systems that we studied. The resulting values of $\tilde{b}$ and $\tilde{c}$ for each of the systems are listed in Table [1]. A universal value of $\tilde{b}=0.412 \pm 0.002$ is obtained from these results.

\section{CRITICAL CROSSING PROBABILITY $\pi_{V}$}

Our simulations for $n_{c}$ could also be used to obtain $\pi_{v}$ by comparing the distance from the growth plane to the maximum height plane. If this distance is greater than or equal to some fixed value $L^{\prime}$, then crossing will occur in an $L \times L \times L^{\prime}$ system (with periodic boundary conditions in each $L \times L$ plane). In other words, we could determine $\pi_{v}\left(L, L, L^{\prime}\right)$ for all $L^{\prime}$ by keeping track of the distribution of distances between the growth plane and maximum height planes in our continuous simulations.

In $2 \mathrm{~d}$, the probability of crossing a system of aspect ratio $r=$ height/width in the vertical direction, with periodic boundary conditions in the horizontal direction, is given by [13,29]

$$
\pi_{v}(r) \sim e^{-2 \pi r(2-D)}=e^{-\frac{5}{24} \pi r}
$$

for large $r$, where $D=91 / 48$ is the 2 d fractal dimension. Eq. (7) follows from a conformal transformation from an annulus to a rectangle, using that the probability a cluster extends 
beyond a radial distance $R$ scales as $R^{D-d}$. We have separately verified that Eq. (7) holds accurately for all $r$ somewhat greater than 1 .

For $3 \mathrm{~d}$ systems, while it still is true that the radial probability scales as $R^{D-d}$, we cannot connect it to $\pi_{v}$ of the $L \times L \times L^{\prime}$ system, because we cannot make a conformal transformation between the concentric spheres and a rectangular solid. However, we still expect an exponential dependence upon $r=L^{\prime} / L$, because that term represents the smallest eigenvalue of the transfer matrix. We thus hypothesize

$$
\pi_{v} \sim K e^{-m r} .
$$

for large $r$. To check this, we plot $\ln \pi_{v}$ vs. $r$ in Fig. 5, which contains the results from all four systems studied, for $L=8,10$, and 12 . To get the best data collapse, we defined $r=\left(L^{\prime}+\ell\right) / L$, which allows for a lattice finite-size effect or boundary extrapolation length in the $L^{\prime}$ direction, in which the effective location of the free boundary is not uniquely defined [22]. (Such an ambiguity in size does not occur in the $L$ directions, because of the periodic boundary conditions.) In fact, the data for all three bond percolation systems collapsed nicely with $\ell=-1.3$, while the data for site percolation on the f.c.c. lattice required a constant of $\ell=1.36$ to fall on the same curve. Figure 6 shows the effect of $\ell$ by comparing an enlarged portion of our data from the s.c. (bond) lattice when $\ell=0$ and $\ell=-1$.3. The corresponding values of $m$ and $\ln K$ are $-1.37 \pm 0.01$ and $0.75 \pm 0.05$, respectively.

\section{DISCUSSION OF RESULTS}

Our values for the critical thresholds of site percolation on the f.c.c. and b.c.c. lattices are listed in Table $\square$. Along with the other results which are summarized in that table, the thresholds of all three $3 \mathrm{~d}$ systems, for both site and bond percolation, are now known to a very high accuracy.

Table $\mathbb{M}$ lists $n_{c}, \tilde{b}$ and $\tilde{c}$ for the four systems studied. Our simulations confirm that $\tilde{b}$ is universal in $3 \mathrm{~d}$ as it is in $2 \mathrm{~d}[2]$, with a value $\tilde{b} \approx 0.412$. In $2 \mathrm{~d}$, the corresponding value is $\tilde{b}=5 \sqrt{3} / 24=0.360844 \ldots$ [ 8 . 
The average density of clusters per site, $n_{c}$, varies from system to system, as expected.

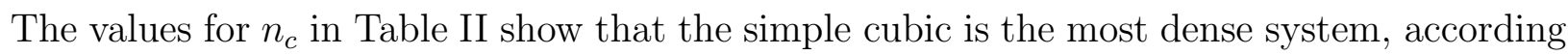
to the convention we used to define the unit volume of the system.

Our simulations have also shown that $\pi_{v}$ is universal as shown in Figure 5, and possesses an exponential decay (8) with $m=1.37 \pm 0.01$, compared with a value of $5 \pi / 24=0.654498 \ldots$ in 2 d. For a cubical system $(L \times L \times L$ or $r=1)$, Eq. (8) implies a value of $\pi_{v}=0.54 \pm 0.04$, while a direct analysis of our data at that point yields the more precise value $\pi_{v}=0.573 \pm 0.005$. The latter value is somewhat higher than the result $0.513 \pm 0.005$ recently reported by Acharyya and Stauffer [21] for a system with helical boundary conditions in the plane, which are similar to periodic boundary conditions but with the rows shifted by one. We believe that in the limit of large $L$ these two boundary conditions should be equivalent, although this belief is not supported by the discrepancy in the values seen above.

Many additional questions are raised for $3 \mathrm{~d}$ systems. What is $b\left(r^{\prime}, r^{\prime \prime}\right)$ where $r^{\prime}=L^{\prime} / L$ and $r^{\prime \prime}=L^{\prime \prime} / L$ for an $L \times L^{\prime} \times L^{\prime \prime}$ system (with periodic boundary conditions in all directions)? What is the effect of helicity or a twist of the order $L$ in the periodic boundary

conditions? Is $\tilde{b}$ related to the number of "percolating" clusters per unit length (however precisely that may be defined)? Finally, can one devise a system that conformally transforms to concentric spheres, so that the crossing probability across that system will be given by a formula analogous to (7)?

\section{ACKNOWLEDGMENTS}

This material is based upon work supported by the US National Science Foundation under Grant No. DMR-9520700. Dietrich Stauffer is thanked for useful comments.

\footnotetext{
\#Electronic mail: cdl@engin.umich.edu

${ }^{\dagger}$ Electronic mail: rziff@engin.umich.edu
} 


\section{REFERENCES}

[1] See, for example, D. Stauffer and A. Aharony, An Introduction to Percolation Theory, Revised 2nd. Ed. (Taylor and Francis, London, 1994); B. D. Hughes, Random Walks and Random Environments (Oxford, London, 1996); A. Bunde and S. Havlin, Fractals and Disordered Systems (Springer, Berlin-Heidelberg, 1996).

[2] R. M. Ziff, S. R. Finch, and V. S. Adamchik, Phys. Rev. Lett., 793447 (1997).

[3] H. N. V. Temperley and E. H. Lieb, Proc. R. Soc. Lond. A. 322, 251 (1971).

[4] R. J. Baxter, H. N. V. Temperley, and S. E. Ashley, Proc. R. Soc. Lond. A. 358, 535 (1978).

[5] V. Privman and M. E. Fisher, Phys. Rev. B 30, 322 (1984).

[6] A. Aharony and D. Stauffer, J. Phys. A: Math Gen. 30, L301 (1997).

[7] B. Müller, Int. J. Mod. Phys. C 9, 1 (1998).

[8] P. Kleban and R. M. Ziff, Phys Rev. B 57, R8075 (1998).

[9] C. D. Lorenz and R. M. Ziff, to be published (1998).

[10] R. Langlands, P. Pouiliot, and Y. Saint-Aubin, Bull. AMS 30, 1 (1994); R. P. Langlands, C. Pichet, P. Pouliot, and Y. Saint-Aubin, J. Stat. Phys. 67, 553 (1992).

[11] J. L. Cardy, J. Phys. A: Math Gen. 25, L201 (1992).

[12] R. M. Ziff, Phys. Rev. Lett.69 2670 (1992).

[13] M. Aizenman, in The IMA Volumes in Mathematics and its Applications (SpringerVerlag, 1997).

[14] G. M. T. Watts, J. Phys. A: Math Gen. 29, L363 (1996).

[15] J.-P. Hovi and A. Aharony, Phys. Rev. E 53, 235 (1996). 
[16] C.-K. Hu, C.-Y. Lin and J.-A. Chen, Phys. Rev. Lett. 75, 193 (1995). C.-K. Hu and J.-A. Chen, J. Phys. A: Math Gen. 28, L73 (1995).

[17] H.-P. Hsu, M.-C. Huang, and K.-J. Ling, Phys. Rev. B 56, 10743 (1997). M.-C. Huang and H.-P. Hsu, J. Phys. A: Math Gen. 31, 3429 (1988).

[18] U. Gropengiesser and D. Stauffer, Physica 210A, 317 (1994).

[19] T. Vicsek and J. Kertész, Phys. Lett. 81A, 51 (1981).

[20] D. Stauffer, J. Adler and A. Aharony, J. Phys. A: Math Gen. 27, L475 (1994).

[21] M. Acharyya and D. Stauffer, Int. J. Mod. Phys. C 9, xxx (1998).

[22] R. M. Ziff, Phys. Rev. E 54, 2547 (1996).

[23] C. D. Lorenz and R.M. Ziff, Phys. Rev. E 57, 230 (1998).

[24] R. M. Ziff, P.T. Cummings, and G. Stell, J. Phys. A: Math Gen. 17, 3009 (1984).

[25] M. E. Fisher, Physics (Long Island City, NY) 3, 255 (1967).

[26] R. M. Ziff and G. Stell, University of Michigan Report No. 88-4, (1988).

[27] H. G. Ballesteros, L. A. Fernández, V. Martín-Mayor, A. Muñoz Sudupe, G. Parisi, and J. J. Ruiz-Lorenzo, preprint (1998), cond-mat/9805125.

[28] P. L. Leath, Phys. Rev. B 14, 5046 (1976).

[29] J. Cardy, J. Phys. A: Math Gen. 31, L105 (1998).

[30] S. C. van der Marck, Phys. Rev. E 55, 1514 (1997).

[31] J. Adler, Y. Meir, A. B. Harris and A. Aharony, Phys. Rev. B 41, 9183 (1990).

[32] P. Grassberger, J. Phys. A: Math Gen. 19, L241 (1986).

[33] P. Grassberger, J. Phys. A: Math Gen. 25, 5867 (1992). 
[34] N. Jan and D. Stauffer, Int. J. Mod. Phys. C 9, 341 (1998). 


\section{FIGURES}

FIG. 1. Plot of $s^{\tau-2} P(s, p)$ vs. $s^{\sigma}$ for (a) f.c.c. and (b) b.c.c. lattices using $\tau=2.189$ and $\sigma=0.445$. Each curve represents a different value of $p$, which are (from top to bottom) (a) $0.1992375,0.1992365$, and 0.1992355 , and (b) 0.245962 5, 0.245 961 5, and 0.2459605.

FIG. 2. Representative $4 \times 4$ planes for the (a) s.c., (b) f.c.c., and (c) b.c.c. lattices. The darkened circles represent active sites in the plane and empty circles represent active sites in the neighboring planes. The solid lines represent bonds in the plane and dashed lines represent bonds which go to the neighboring planes.

FIG. 3. Plot of $n$ vs. $1 / L^{3}$ for bond percolation on the b.c.c. lattice. The intercept of this plot yields $n_{c}$ and the slope yields $\tilde{b}$ according to Eq. (5).

FIG. 4. Plot of $\left(n-n_{c}\right) L^{3}$ vs. $1 / L^{3}$ for the b.c.c. (bond), f.c.c. (bond), f.c.c. (site), and s.c. (bond) (from top to bottom) systems at $p_{c}$. In these plots, the intercept represents the value of $\tilde{b}$ and the slope is the second correction term $\tilde{c}$. The values of $\tilde{b}$ and $\tilde{c}$ are listed in Table II.

FIG. 5. Plot of $\ln \pi_{v}$ vs. $r=\left(L^{\prime}+\ell\right) / L$ for the b.c.c. (bond) (dashed lines), f.c.c. (bond) (dotted lines), f.c.c. (site) (also dotted lines), and s.c. (bond) (solid lines) lattices of size $L \times L \times L^{\prime}$ with $L=8,10$, and 12 at $p_{c}$. In these plots, the intercept represents the value of $\ln K$ and the slope is $m$. The values of $\ln K$ and $m$ are $0.75 \pm 0.05$ and $-1.37 \pm 0.01$.

FIG. 6. Plot of $\ln \pi_{v}$ vs. $r=\left(L^{\prime}+\ell\right) / L$ for an enlarged portion of the data from the s.c. (bond) lattice of size $L \times L \times L^{\prime}$ with $L=8$ (square), 10 (circle), and 12 (triangle) at $p_{c}$. The upper three curves show the data plotted with $\ell=0$, and the bottom curve shows the data collapse when $\ell=-1.3$ is used. 


\section{TABLES}

TABLE I. Values of $p_{c}$ for bond and site percolation on the b.c.c., f.c.c. and s.c. lattices from present $\left(^{*}\right)$ and other recent work. The numbers in parenthesis represent the errors in the last $\operatorname{digit}(\mathrm{s})$.

\begin{tabular}{|c|c|c|c|}
\hline system & $p_{c}$ & Ref. & Value used here \\
\hline \multirow[t]{3}{*}{ b.c.c. (bond) } & 0.1803 & 迎 & \\
\hline & $0.1802(2)$ & 30 & \\
\hline & $0.1802875(10)$ & 23 & 0.1802875 \\
\hline \multirow[t]{3}{*}{ b.c.c. (site) } & 0.246 & 1] & \\
\hline & $0.2458(2)$ & 30 & \\
\hline & $0.2459615(10)$ & $*$ & \\
\hline \multirow[t]{3}{*}{ f.c.c. (bond) } & 0.119 & 1] & \\
\hline & $0.1200(2)$ & 30 & \\
\hline & $0.1201635(10)$ & 23 & 0.1201635 \\
\hline \multirow[t]{3}{*}{ f.c.c. (site) } & 0.198 & $1]$ & \\
\hline & $0.1994(2)$ & 30 & \\
\hline & $0.1992365(10)$ & $*$ & 0.1992365 \\
\hline \multirow[t]{7}{*}{ s.c. (bond) } & 0.2488 & [1] & \\
\hline & $0.2487(2)$ & 30 & \\
\hline & $0.2488(2)$ & 31 & \\
\hline & $0.24875(13)$ & 32 & \\
\hline & $0.248814(3)$ & 33 & \\
\hline & $0.248812(2)$ & 26 & \\
\hline & $0.2488126(5)$ & 23 & 0.2488126 \\
\hline \multirow[t]{3}{*}{ s.c. (site) } & 0.3116 & [1] & \\
\hline & $0.3114(4)$ & 30 & \\
\hline & $0.311605(10)$ & 26 & \\
\hline
\end{tabular}




\begin{tabular}{ll}
$0.311604(6)$ & 33 \\
$0.311605(5)$ & 21 \\
$0.311600(5)$ & 27 \\
$0.3116081(13)$ & 9 \\
$0.3116080(4)$ & 9 \\
\hline
\end{tabular}

TABLE II. Values of $n_{c}$ (clusters per unit volume), $\tilde{b}$, and $\tilde{c}$ for the systems studied.

\begin{tabular}{lllr}
\hline \hline system & $n_{c}$ & $\tilde{b}$ & $\tilde{c}$ \\
\hline \hline s.c. (bond) & $0.2729310(5)$ & $0.414(3)$ & $6.0(7)$ \\
\hline f.c.c. (bond) & $0.1538440(5)$ & $0.414(3)$ & $-1.4(3)$ \\
f.c.c. (site) & $0.0132655(5)$ & $0.409(3)$ & $-1.8(3)$ \\
\hline b.c.c. (bond) & $0.0745860(5)$ & $0.409(3)$ & $-5.5(7)$ \\
\hline \hline
\end{tabular}


Figure 1(a)

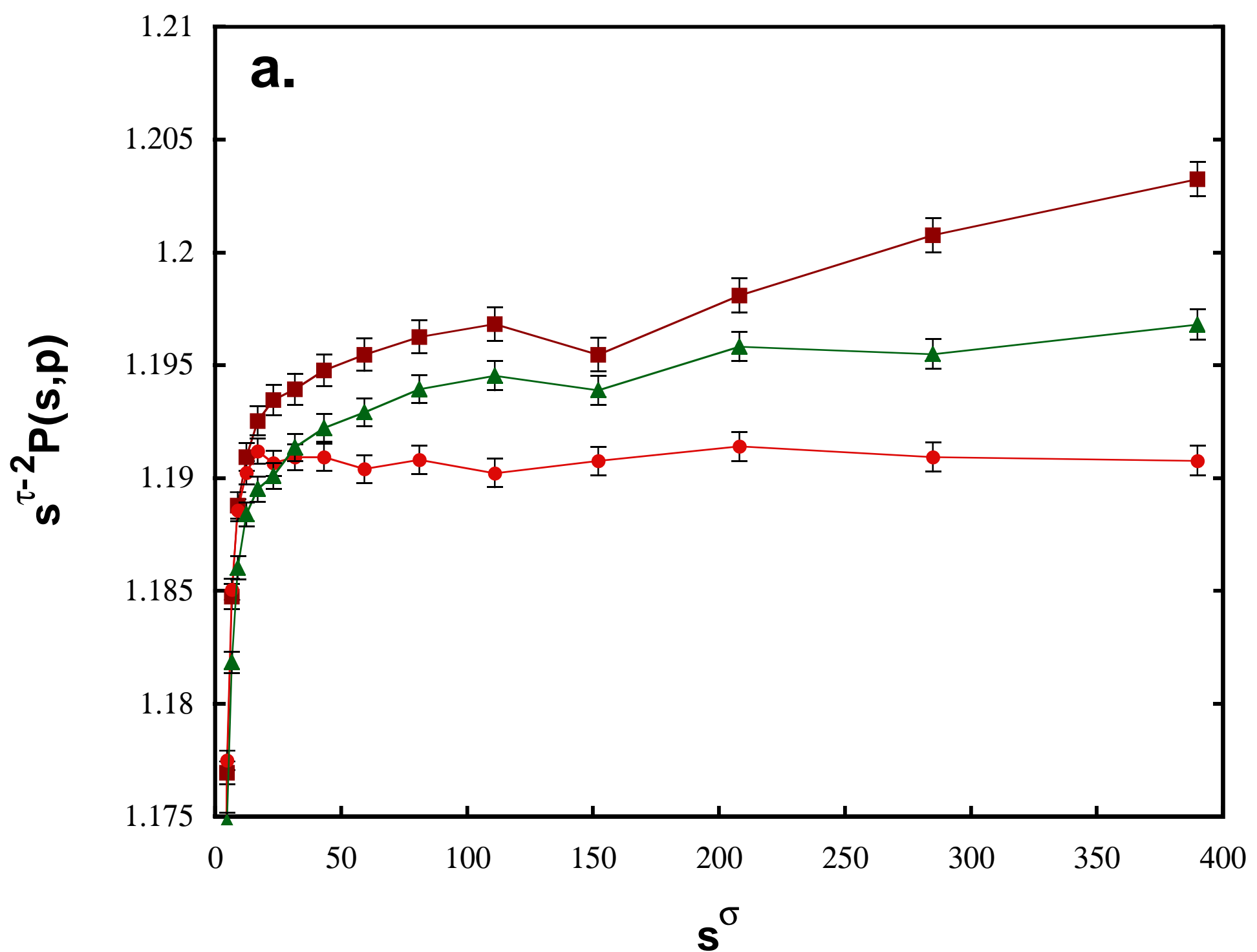


Figure 1(b)

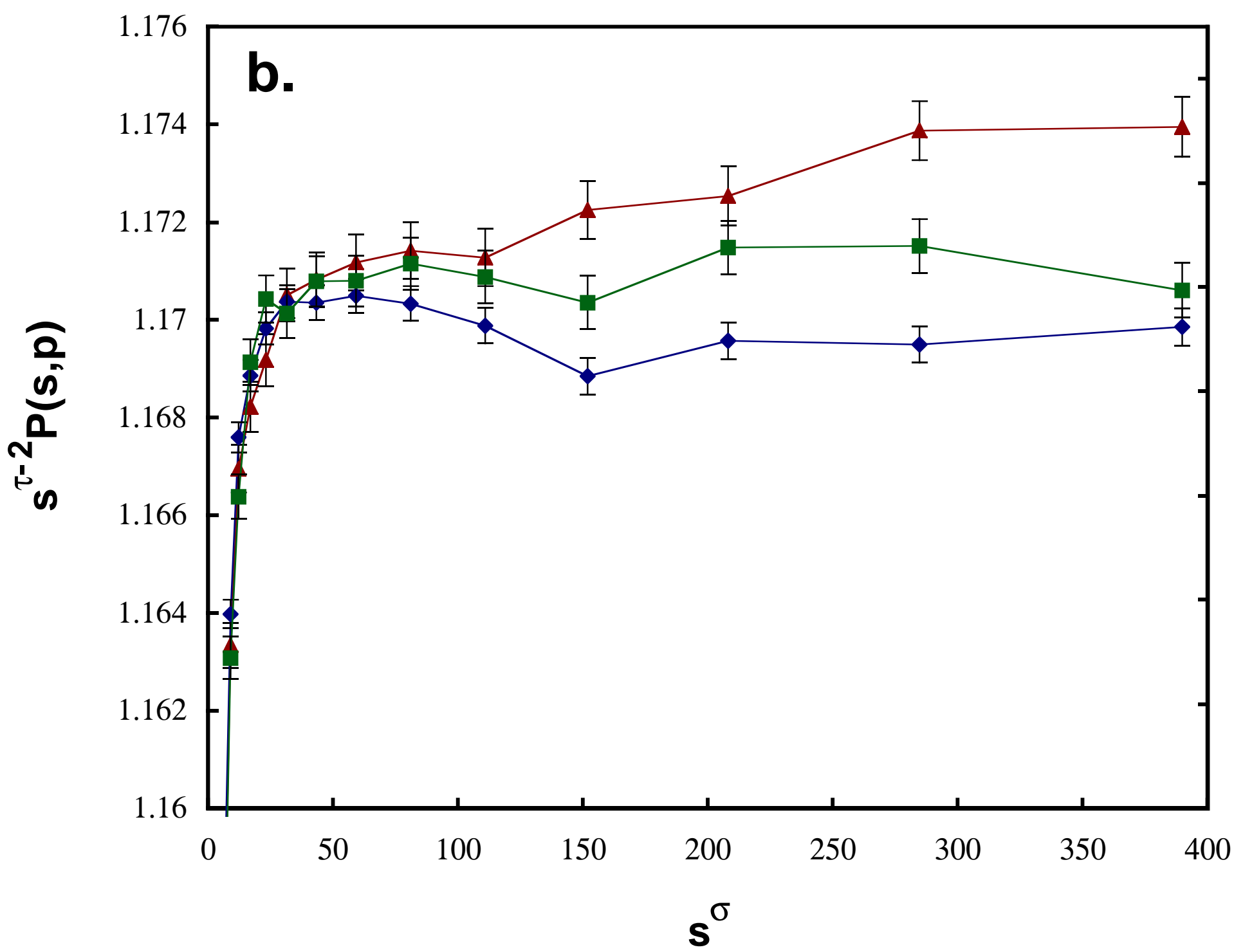




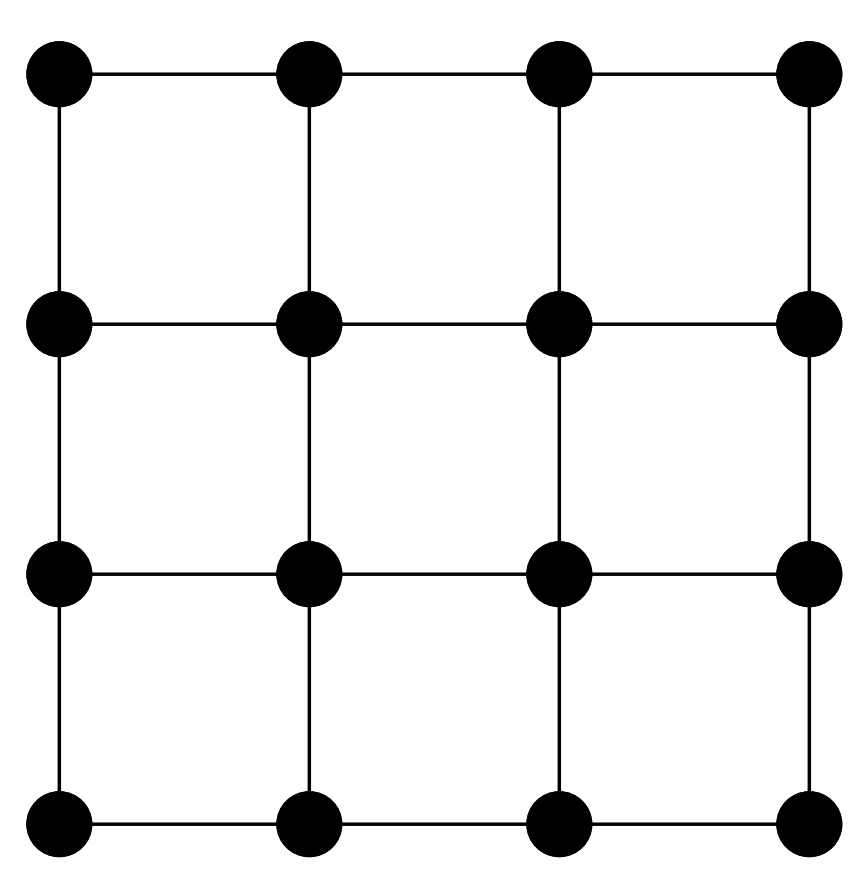

a
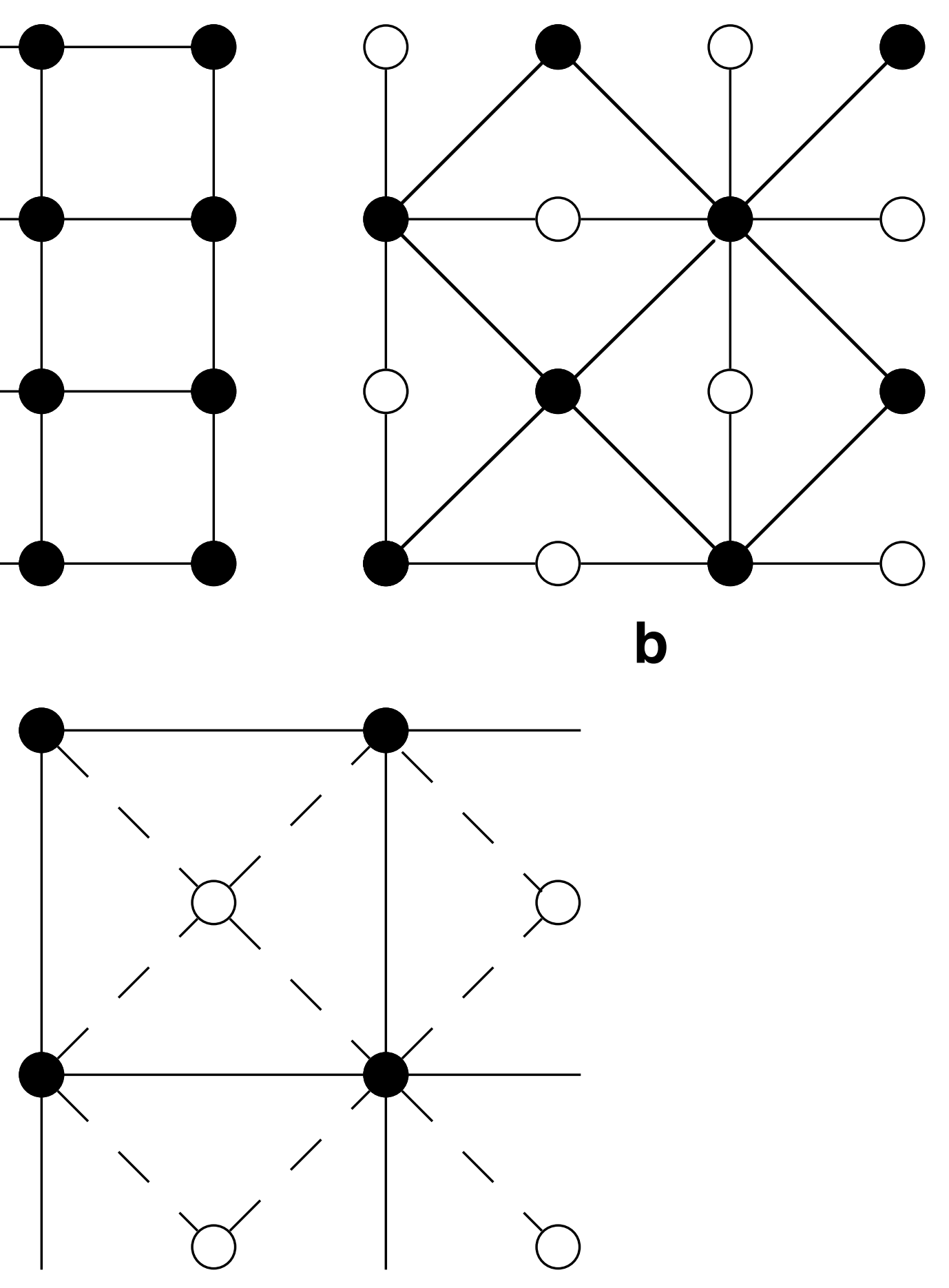

C 
Figure 3

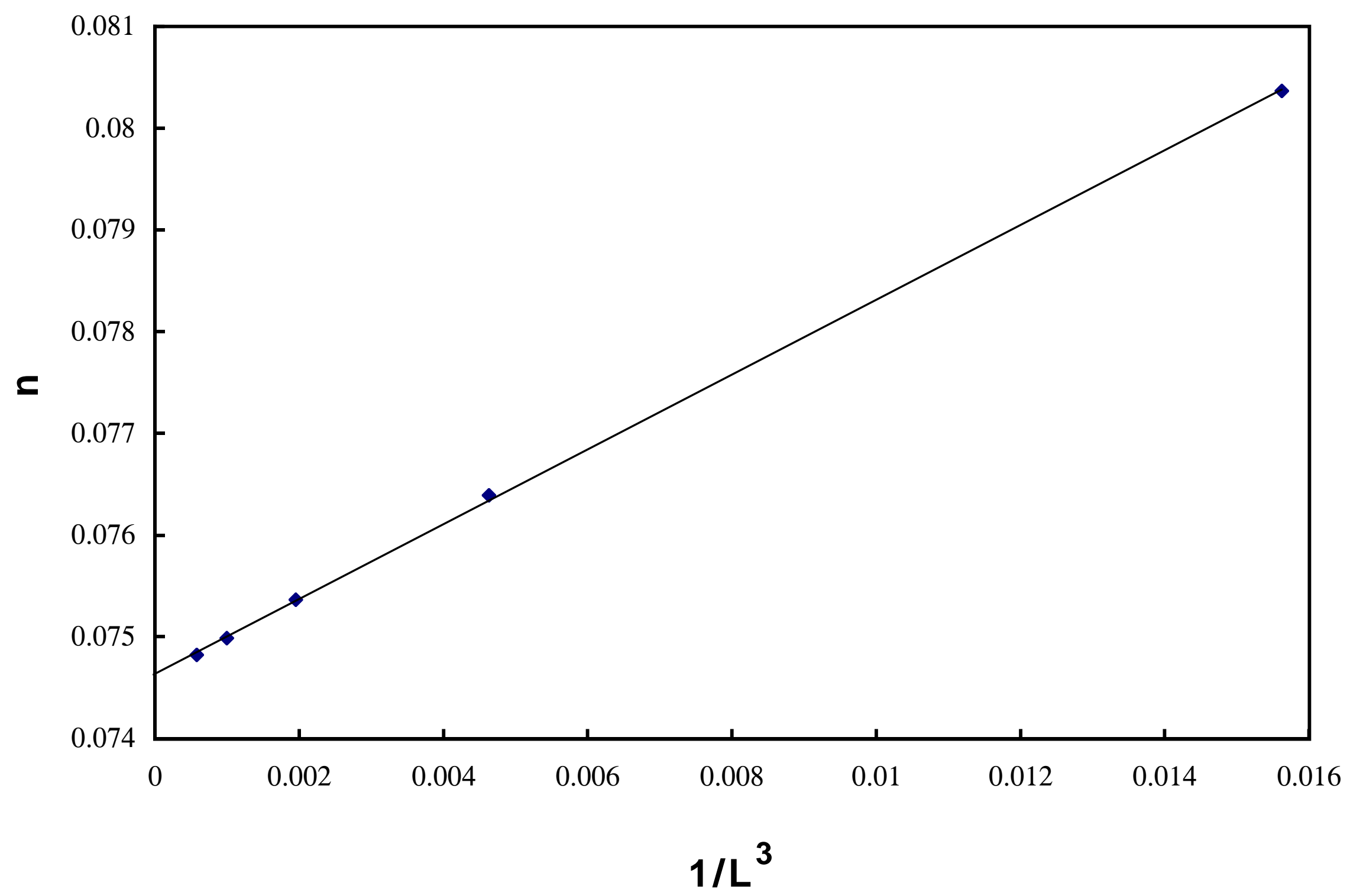


Figure 4

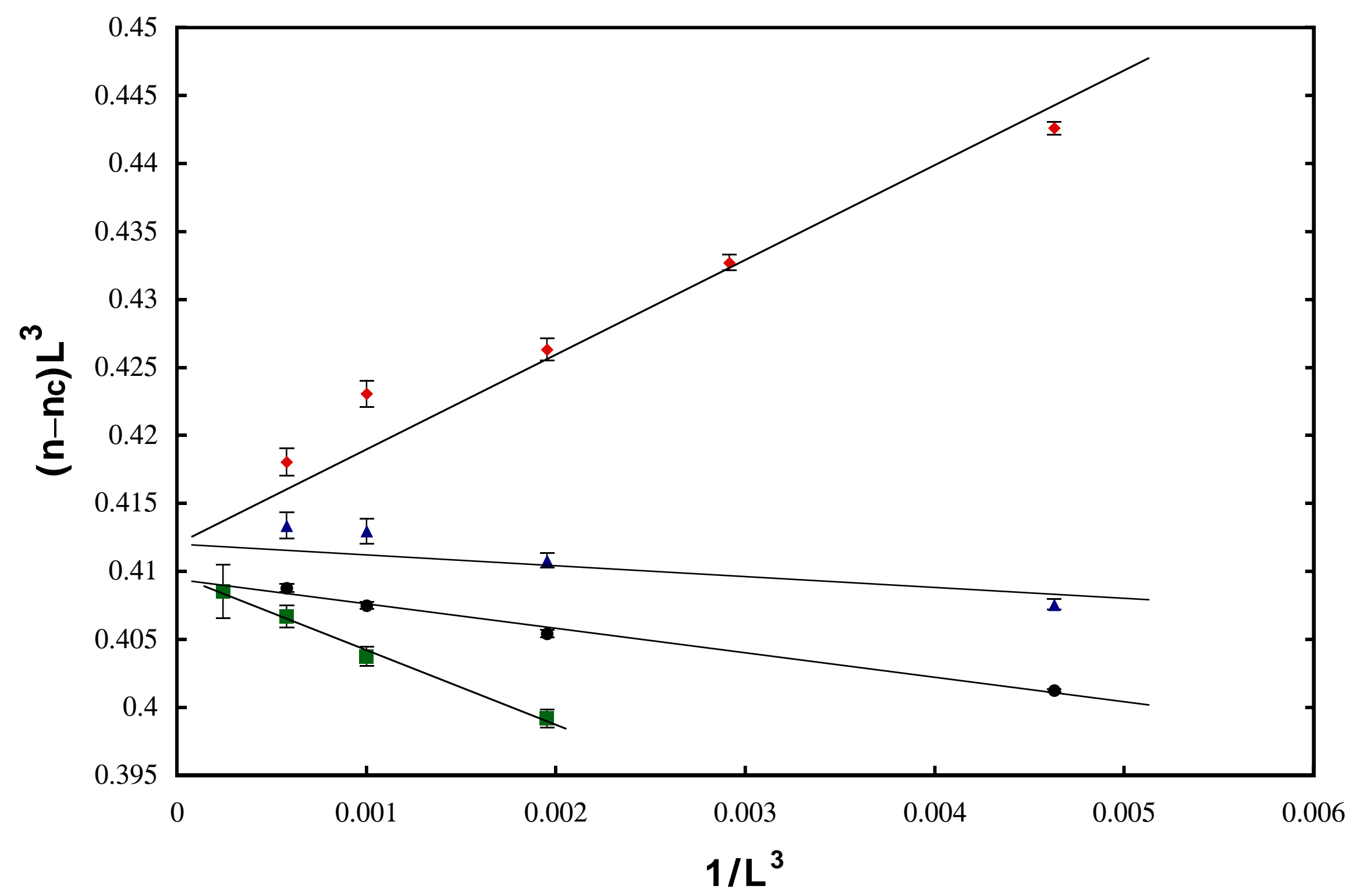


Figure 5

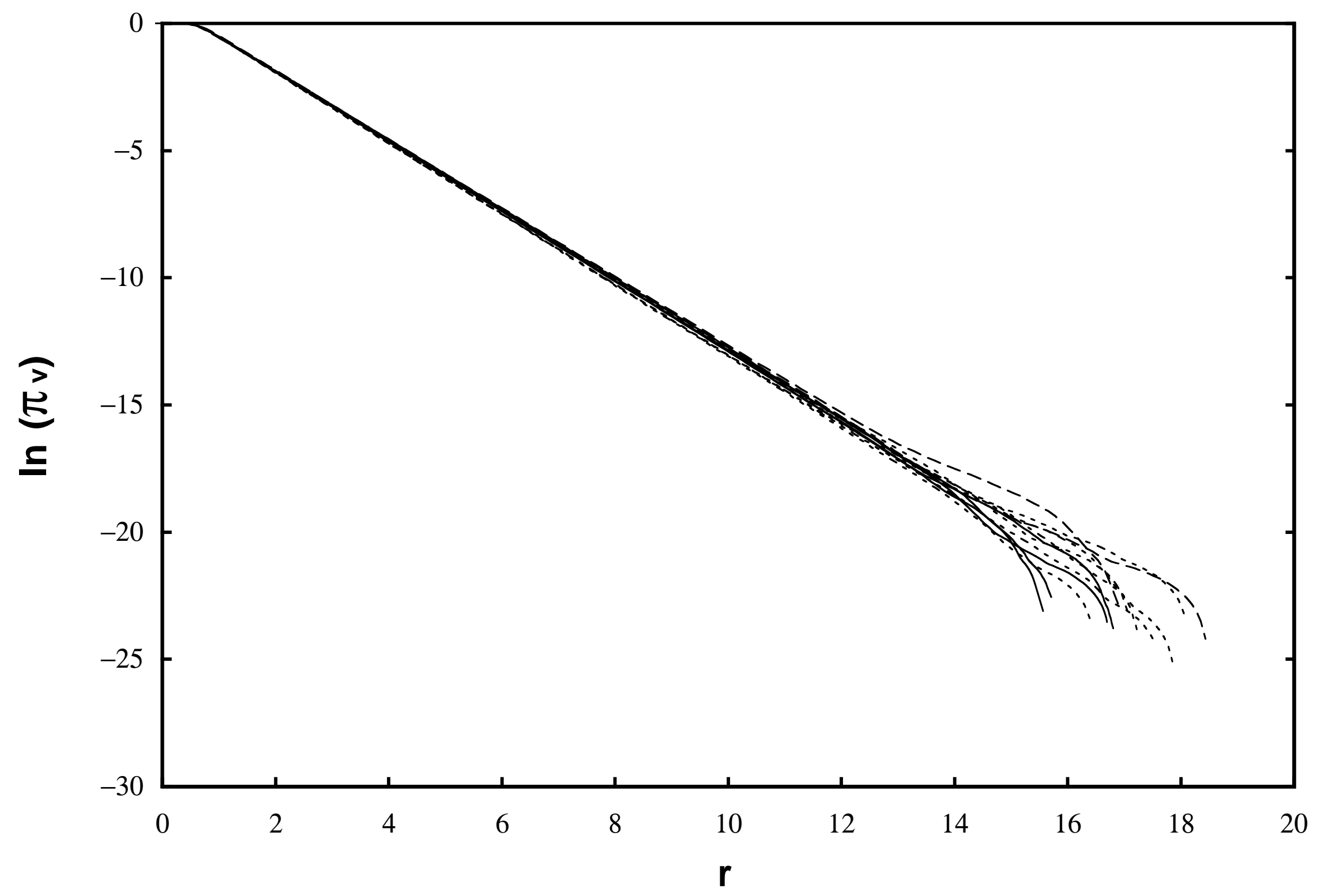


Figure 6

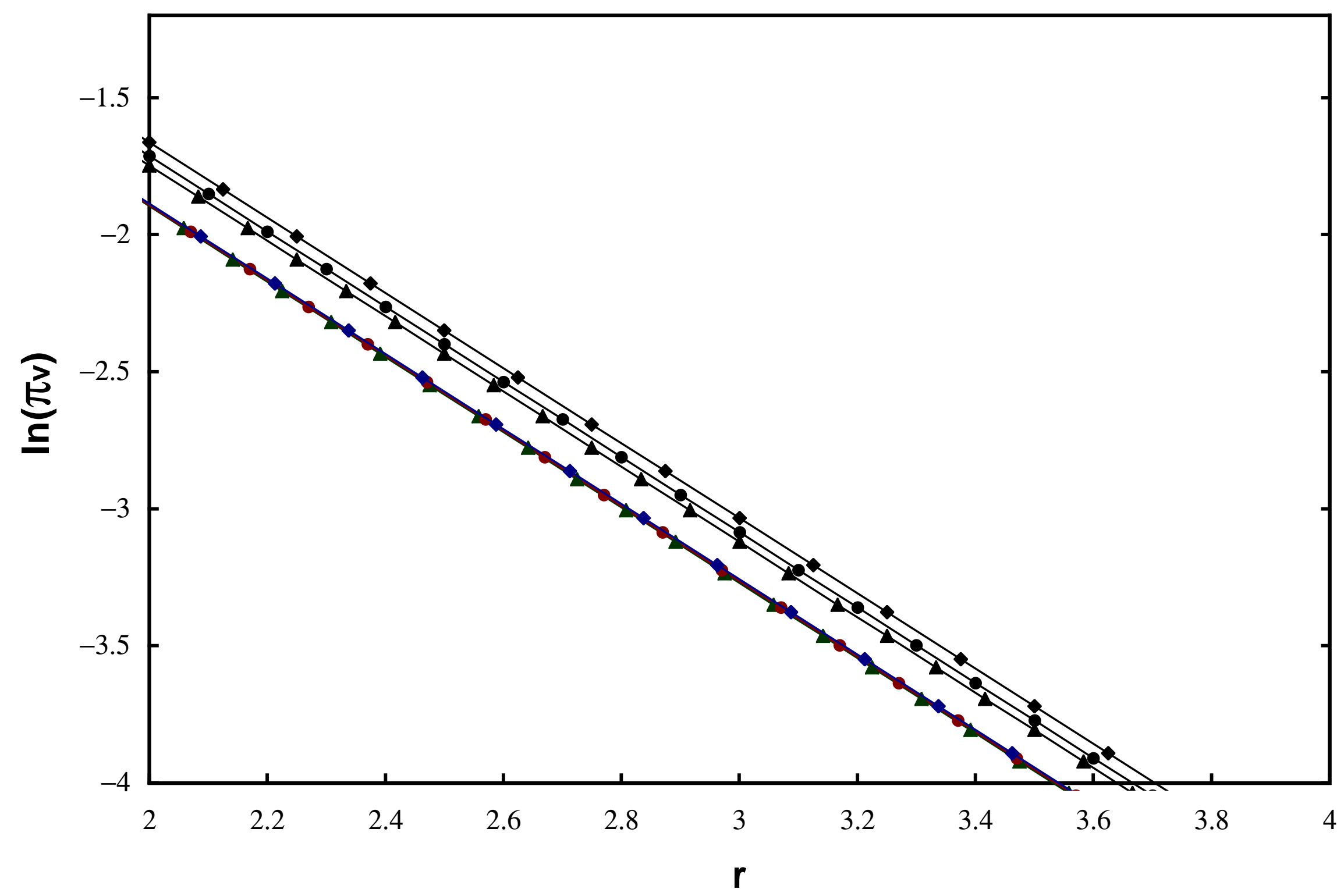

